\title{
Uncertainty Investigation for Personalised Lifelogging Physical Activity Intensity Pattern Assessment with Mobile Devices
}

\author{
Jun Qi, Po Yang, Martin Hanneghan, Kieran Latham, Stephen Tang \\ Department of Computer Sciences \\ Liverpool John Moores University \\ Liverpool, UK, L3 3AF \\ j.qi@2015.ljmu.ac.uk
}

\begin{abstract}
Lifelogging physical activity (PA) assessment is crucial to healthcare technologies and studies for the purpose of treatments and interventions of chronic diseases. Traditional lifelogging PA monitoring is conducted in nonnaturalistic settings by means of wearable devices or mobile phones such as fixed placements, controlled durations or dedicated sensors. Although they achieved satisfactory outcomes for healthcare studies, the practicability become the key issues. Recent advance of mobile devices make lifelogging PA tracking for healthy or unhealthy individuals possible. However, owning to diverse physical characteristics, immaturity of PA recognition techniques, different settings from manufactories and a majority of uncertainties in real life, the results of $\mathrm{PA}$ measurement is leading to be inapplicable for PA pattern detection in a long range, especially hardly exploited in the wellbeing monitoring or behaviour changes. This paper investigates and compares uncertainties of existing mobile devices for individual's PA tracking. Irregular uncertainties (IU) are firstly removed by exploiting Ellipse fitting model, and then monthly density maps that contain regular uncertainties $(\mathrm{RU})$ are constructed based on metabolic equivalents (METs) of different activity types. Five months of four subjects PA intensity changes using the mobile app tracker Moves [1] and Google Fit app on wearable device Samsung wear S2 are carried out from a mobile personalised healthcare platform MHA [2]. The result indicates that uncertainty of PA intensity monitored by mobile phone is $90 \%$ lower than wearable device, where the datasets tend to be further explored by healthcare/fitness studies. Whilst PA activity monitoring by mobile phone is still a challenging issue by far due to much more uncertainties than wearable devices.
\end{abstract}

Index Terms-physical activity, intensity pattern, ellipse fitting model, density map, mobile device.

\section{INTRODUCTION}

L Jifelogging physical activity (PA) aims at capturing one's entire life using digital devices for health statues and wellness. Thus as a key role plays in a number of chronic diseases, its effectiveness and accuracy are critical to conduct personalised clinical diagnoses of treatments and preventions $[3,4]$. Normally, PA assessment in clinics filed are mostly observed either through distributing several wearable sensors over one's body and training their sensory data with advanced machine learning algorithms [5-9], or capturing and analysing a series of images with wearable cameras $[10,11]$. Both ways exhibits relatively accurate measures, however, their obtrusiveness, cost, battery life and storage capacity are the serious issues leading to the limitation of permanently applying to the completely natural environments.

In recent years, the progress of mobile devices with embedded inertial sensors are generating increasing public attention. Popular products, such as Fitbit Flex [12], Nike+ Fuelband [13], JawboneUp [14], etc., are wristband devices that record PA information (e.g. steps, distance, and calories burnt) and other physiological information (e.g. heartbeat rate). Mobile apps, such as Moves [1] and Google Fit [15] are based on smartphone 3D accelerometer data and GPS information which allows tracking the user's movements including location, distance and speed.

With the pervasive utilization of heterogeneous sensors (e.g., accelerometers, gyroscopes, altimeters, temperature, pressure) significant advancements in smart healthcare have generated a large amount of opportunities in industrial areas [16-18], particularly in healthcare field [18-20]. Due to the exponential growth of commercial wearable devices and mobile apps, it has become increasingly possible to remotely monitor and measure PA data by connecting heterogeneous medical devices into a smart healthcare platform [21]. Unfortunately though, due to the commercial perspective, nearly all of the popular wearable devices and mobile apps in the market focus more on personal fitness and exhibit a lack of compatibility and extensibility [22,23]. Also, owing to the heterogeneity of connected devices and rapid change of diverse life patterns in an IoT environment, lifelogging PA information captured by mobile devices usually contains much uncertainty. Effective validation of these high volume and multi-dimensional lifelogging PA data becomes an extremely difficult task. Traditional PA validation methods hardly deal with these scattered and heterogeneous data sets. In the existing literature, none is reported to successfully improve the accuracy of these wrapped PA data collected by mobile devices in a smart healthcare environment.

Our study in this paper attempts to take lifelogging PA as a target to explore the possibility of utilizing new techniques for investigating and comparing the feasibilities of customer device/app for lifelogging PA assessment in a smart healthcare environment. We first give a comprehensive review of existing life-logging PA measurement mobile devices, and identify regular (RU) and irregular (IU) uncertainties of these life-logging PA measures in a smart healthcare environment. We use Ellipse fitting model to remove RU, and make density map for each month with an individual's PA intensity using mobile phone and wearable device respectively. PA intensity of four subjects with two devices are analysed and compared from the mobile personalised healthcare platform MyHealthAvatar (MHA) [2] in our case study. 
The rest of the paper is structured as follows. Section II presents the literature review of related work. Section III describes the Ellipse fitting model for removing IU and the density map constructions and RU analysis. Section IV reports a case study for evaluating datasets from mobile phone and wearable device in MHA platform. Finally, the conclusions and future work are presented in Section V.

\section{RELATED WORK}

PA intensity based on the energy expenditure is categorised into four types: sedentary (e.g., sitting and lying), light (e.g., standing, desk job, etc.), moderate (e.g., walking, cycling, etc.), vigorous (jogging, swimming etc.) and high (e.g., fast running, weightlifting, etc.). Traditionally, PA is mostly observed based on accelerometer technology as well as easily and openly accessible Global Position System (GPS). Numerous research works and commercial products have attempted to accurately monitor PA and access activity patterns and intensity level, by using either dedicated wearable sensors $[24,25]$ or conducted in controlled or semi-controlled environment. Fig.1 accurately presents the typical daily adult PA intensity pattern. This result comes from accelerometer monitoring from a few studies under such conditions [26].

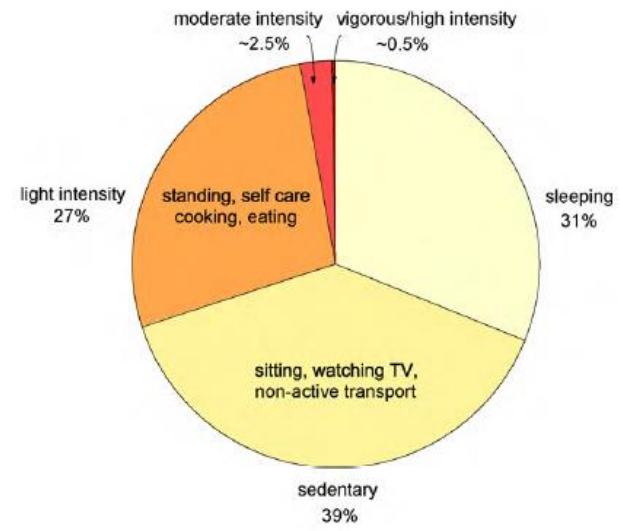

Fig. 1. The typical adult pattern of daily activities [percentage of a 24-h day] when categorised in terms of intensity level assessed using accelerometer counts. About 31\% [7.5 h] is sleep, 39\% [9.4 h] is spent in sedentary activities [sitting], 27\% [6.5 h] in light activities and only about $3 \%$ of the $24-\mathrm{h}$ day [ $43 \mathrm{~min}$ ] is spent in moderate-high intensity activities [26].

Recently, many commercial wearable products and mobile applications have been released that support long term recording and collection of personal health information, particularly on physical activity. Popular mobile apps, such as Moves, are based on smartphone 3D accelerometer data and GPS information which allows tracking user movement activities including location, distance and speed. The wearable products, such as Fitbit Flex, Nike+ Fuelband, JawboneUp, are all wristband devices that record steps count, distance, and calories burnt. However, lifelogging physical activity measure with mobile devices has significant difference with traditional PA measures on four aspects: low accuracy, data encapsulated, long term observation and uncontrolled environments. Thus, these four aspects lead to a variety of uncertainties for lifelogging PA measures.

\section{UNCERTAINTY}

Customer PA monitors like wearable devices and mobile phones have addressed some practical issues such as storage, battery life and cost, especially mobile apps which are often free. Nevertheless, PA recognition results offered by mobile devices are widely divergent as a result of different places being carried by different users such as pocket or handbags. Furthermore, the diverse life pattern of an individual person may cause huge indeterminateness, as they perform PA in varying ways owning to age, gender, weight, etc. Hence, a specific PA tracking model that fits one group of user may not fit another one. In addition to that, some applications often automatically switch off themselves for energy efficiency which has contributed to missing data. In general, the uncertainties of lifelogging PA from customer devices here is divided into two types as our previous work investigated [23][27][28]:

Irregular Uncertainty (IU): randomly and accidently occurs in lifelogging PA data. The causes of these uncertainties include device malfunctions or faults, breakdown of a third party server, misuse of devices or sudden change of personal circumstance. The occurrence of IU will appreciably impact the efficiency and accuracy of assessing personal health.

Regular Uncertainty (RU): frequently and persistently occurs in lifelogging PA data. The causes resulting in these uncertainties are mainly from some regular influencing issues, like intrinsic sensors' errors, differentiation of personal physical fitness and changes of environment. The occurrence of regular uncertainty in physical activity data is inevitable so that it is impossible to completely eliminate these uncertainties.

\section{A. Handling with IU}

The level of PA is assessed and represented by the number of steps walking per day or the distance walking per day. Current mobile devices enable measuring walking speed related information, like Daily Walking Speed. Therefore, our inspiration for managing the above two types of uncertainties is to build a 2D distribution of physical activity regarding two benchmarks: Daily Walking Steps (Steps) and Daily Walking Speed (Speed). Fig. 2 shows a typical raw data distribution. As we can see, some points exceed regular ranges, e.g., 17000 steps/ hour. We use Ellipse fitting model for the removal of IU, where the confidence interval between $95 \%$ and $98 \%$.

In Fig.2, the red dots fall out of the Ellipse circle represents the IU, the hollow dots are the regular physical activity data covered by the Ellipse algorithm. A noticeable issue here is that we only consider the lower limits of walking steps and the upper limits of walking speeds as threshold parameters. On some days users might walk distinctly more steps than usual, while the other days might be more sedentary. 


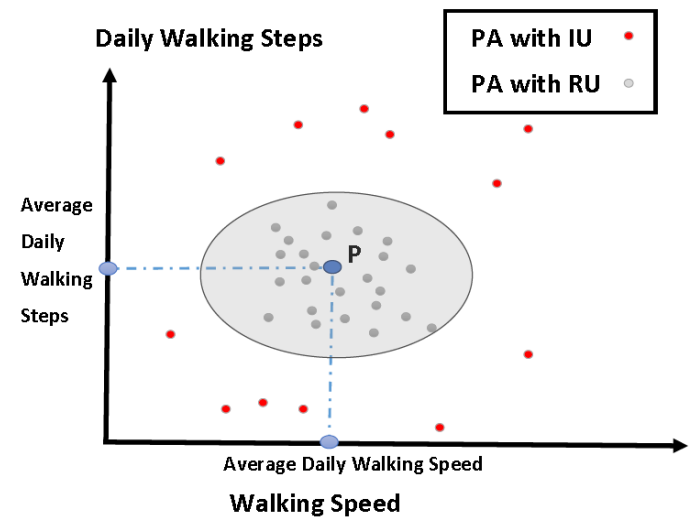

Fig. 2 Distribution of irregular uncertainties and regular uncertainties

\section{B. Density map visualisation}

We also use a density map to construct longitudinal PA monitoring data distribution as our previous work [27]. An example of a density map derived from the mobile device is presented as Fig. 2. Here we select (METs) and PA duration as the validation standard for the each PA intensity. In other words, the intensity of the activity is based on the amount MET $\times$ duration. Some typical intensity levels emerged for all the PA types of the mobile devices are presented in the table 1 .

Table 1. PA intensity levels standard [29]

\begin{tabular}{ll}
\hline PA intensity & MET \\
\hline Writing, desk work, typing & 1.8 \\
\hline Slowing walking $(2.7 \mathrm{~km} / \mathrm{h})$ & 2.3 \\
\hline Normal walking $(4 \mathrm{~km} / \mathrm{h})$ & 2.9 \\
\hline Light cycling $(15 \mathrm{~km} / \mathrm{h})$ & 5.9 \\
\hline Normal cycling $(20 \mathrm{~km} / \mathrm{h})$ & 7.1 \\
\hline Jogging & $7.0-8.0$ \\
\hline Running & $8.0-9.0$ \\
\hline
\end{tabular}

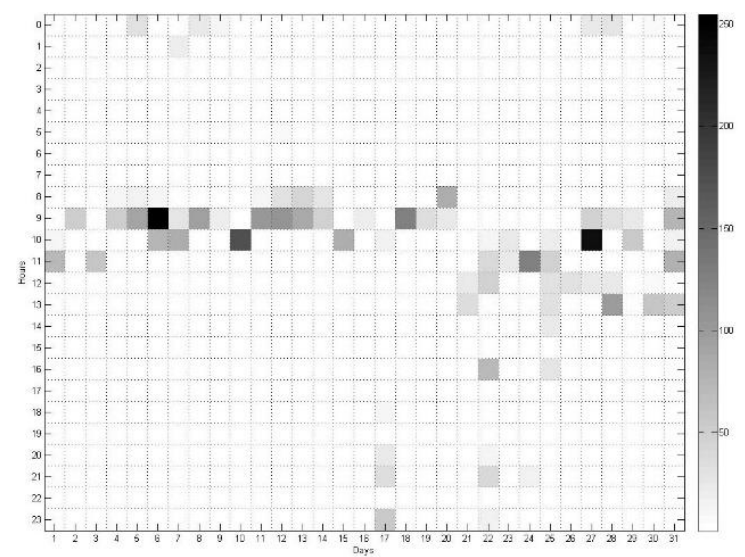

Fig.3. an example of density map of PA intensity from the mobile device's dataset

Intensity $=\frac{\text { ActivityDuration } / h r \times M E T / h r}{\text { maxActivityDuration } / h r \times M E T / h r} \times$ pixel

Where ActivityDuration $/ \mathrm{hr} \times \mathrm{MET} / \mathrm{hr}$ refers to intensity of the PA in each hour; ActivityDuration $/ \mathrm{hr} \times$ $M E T / h r$ refers to maximum intensity value of the month; In the density map, the vertical axis indicates 24 hours a day. While horizontal axis indicates days of each month from 1st to 30th or 31st. Image pixel ranges from 0 to 255 , which means the image is in the grey level. PA intensity ranges from light colour to dark colour, where dark colour denotes very high intensity and vice versa. The white part in the map represents sedentary and uncertain patterns.

\section{Feature extraction}

To measure the dissimilarities of each monthly density map, we select six histogram features (mean, variance, skewness, kurtosis, energy and entropy) from the map. The mean, variance and skewness present the degree of average, discrete and asymmetrical distribution in a grayscale histogram, respectively. Kurtosis measures the relative peakness or flatness of the distribution to a normal distribution. Energy and entropy represent the average degree of gray distribution. We also select four texture features based on gray-level co-occurrence matrix (GLCM) which are contrast, correlation, energy and homogeneity. Contrast measures the local variations in the gray-level cooccurrence matrix. Correlation measures the joint probability occurrence of the specified pixel pairs. Energy provides the sum of squared elements in the GLCM. Also known as uniformity or the angular second moment. Homogeneity measures the closeness of the distribution of elements in the GLCM to the GLCM diagonal.

\section{Distance measure and $R U$ determination}

In order to assess the subject's PA intensity state, Euclidean distance is adopted to measure the dissimilarity among density maps, as Eq. (2).

distance $_{i, j}=\sqrt{\sum_{i=2, j=1}^{k}\left(x_{i, j}-x_{i-1, j}\right)^{2}}$

Where $x$ reprensts the vectors of extracted features. $x=$ $\left[x_{1} \ldots x_{k}\right]$. The smaller the distance, the similar the two map images, and vice versa.

The validation dataset features four randomly selected healthy individuals using the mobile devices for 5 months. The subjects investigated are staff and research students at a university. They are working 6 to 8 hours in front of a computer every work day, whilst PA intensity and time are relatively stable each month.

\section{CASE STUDY: UNCERTAINTY EVALUATION AND COMPARISONS}

In this case study, we create four months density maps from the subject using Moves app on mobile phone and Google Fit app on Samsung Gear S2, respectively, with the datasets from July to October 2015. To measure the uncertainties of each month's PA intensity pattern, histogram and texture features are extracted from both devices. The subject used the mobile app Moves and Google Fit datasets are collected from the mobile personalised healthcare platform MHA [2].

\section{A. Density maps dissimilarity evaluation from mobile phone}

The subject in the case study is a female, 30 years old, working as a researcher at the university 7 hours (desk job), 
and working out 1.5 hours per working day. Four months PA intensity are represented as the density maps shown as fig.4 (a) and fig.5 (a) from the mobile phone and wearable device respectively. Whilst fig.4 (b), (c) and fig.5 (b), (c) show the histogram features and texture features extracted from the maps, representing the PA intensity pattern dissimilarities among the four month using both devices. However, the subject's lifestyles are relatively stable, while as we can see from the dissimilarity measures, the trend line displays large distance changes, which proves that much uncertainty exists using the commercial products.

Compared with mobile phone PA tracker, the wearable device presents relatively more stable and less limit of fluctuations. As we can see in the fig.4 (b) and fig.5 (b), the fluctuation limit is 2.8 with wearable device, while mobile phone is up to 10 , and thus the uncertainty distance among each month is bigger than the wearable device. The key reason is the placement on human's body that the subject is able to take the band on the wrist all the time. Also, due to the battery and capacity saving mode, PA apps on mobile phones switch themselves off occasionally. The mobile device/sensor placement on human body is therefore attracting increasingly attentions in human physical activity research field in recent years [30].

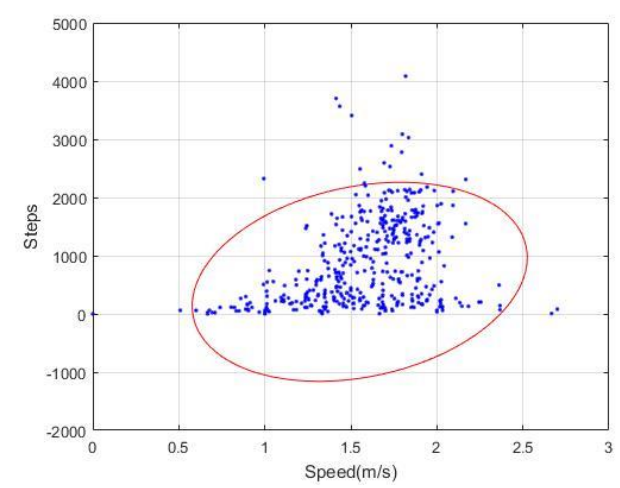

(a)
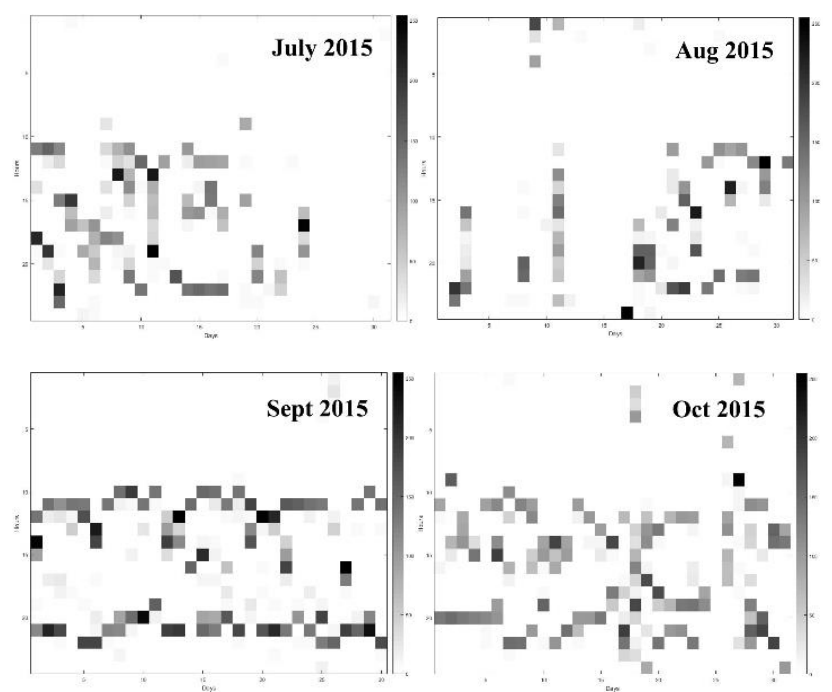

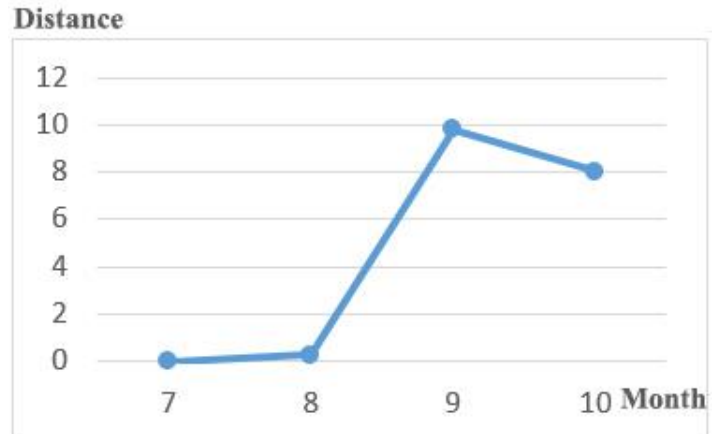

(c)

Fig.4. (a) Ellipse fitting model for IU removal of consecutive four months' of the subject's Moves dataset $(\mathrm{c}=0.98)$ from mobile phone datasets $(b)$ Density maps of four consecutive months' datasets from mobile phone dataset (c) histogram and texture features dissimilarity for four consecutive months' datasets from mobile phone dataset

\section{B. Density maps dissimilarity evaluation from wearable device}

We also collect the Google Fit datasets from wearable device from the same subject. As we can see from the fig.5 (a), average walking/jogging speed is higher than mobile phone, and so are the daily steps. Fig.5 (b) shows more intensive data cells compared with phone's datasets, which denotes that wearable device has relatively stable PA intensity records. The result is also shown in the fig.5 (c) that distances among each monthly density map are lower than the datasets from phone.

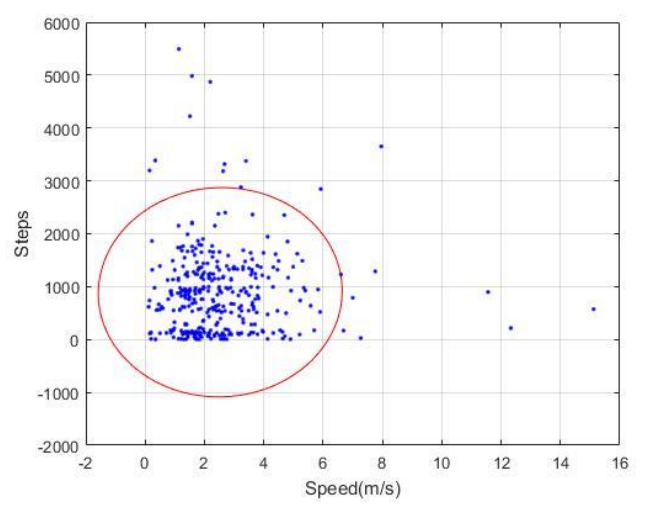

(a)

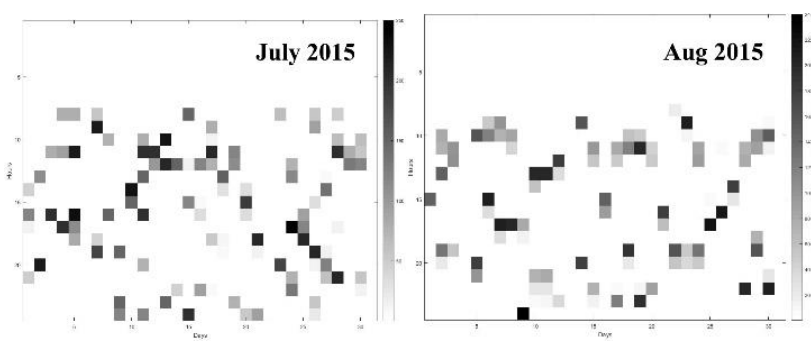




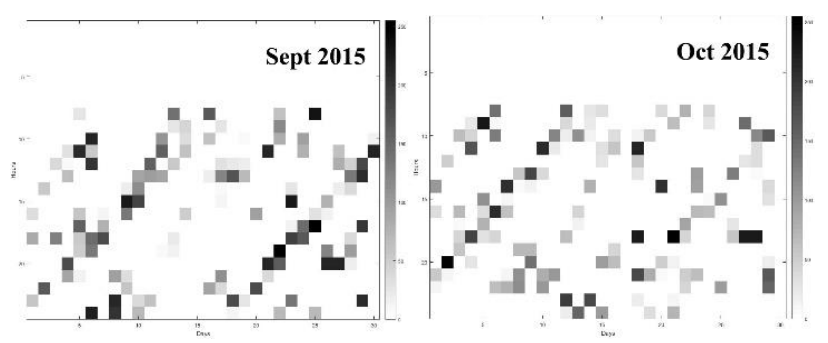

(b)

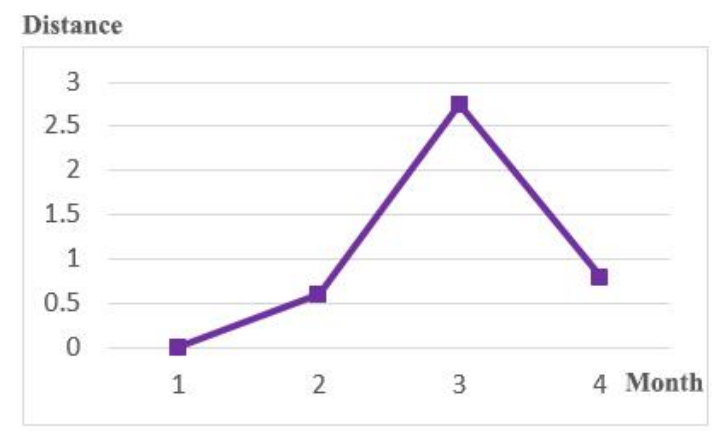

(c)

Fig.5. (a) Ellipse fitting model for IU removal of consecutive four months' of the subject's Moves dataset $(c=0.98)$ from wearable device datasets (b) Density maps of consecutive four months' datasets from wearable device datasets (c) histogram and texture features dissimilarity for four consecutive months' datasets from wearable device datasets

\section{PA Intensity pattern comparisons with two devices}

The devices of mobile phone and wearable device usage of four subjects' PA intensity for consecutive five months are presented in the fig. 6 and fig.7. The subjects are staff and research students at university, spending 6 to 8 hours sitting in front of computer, so the lifestyles are relatively sedentary. Subject 1 has less fluctuations with mobile phone, while others show more uncertainties with both histogram and texture features. On the other hand, the distance of wearable device is $90 \%$ lower than mobile phone, which presents more stable with less uncertainties in lifelogging PA monitoring.

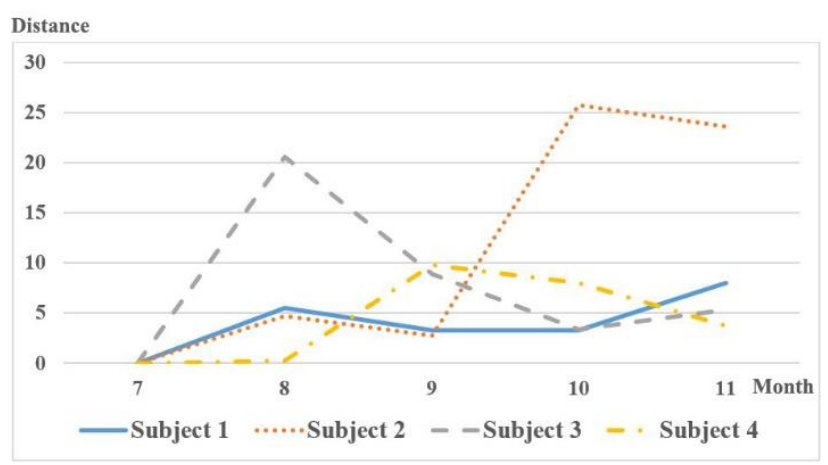

Fig. 6. Four subjects' density map dissimilarities comparison of five months using mobile phone from histogram and texture features

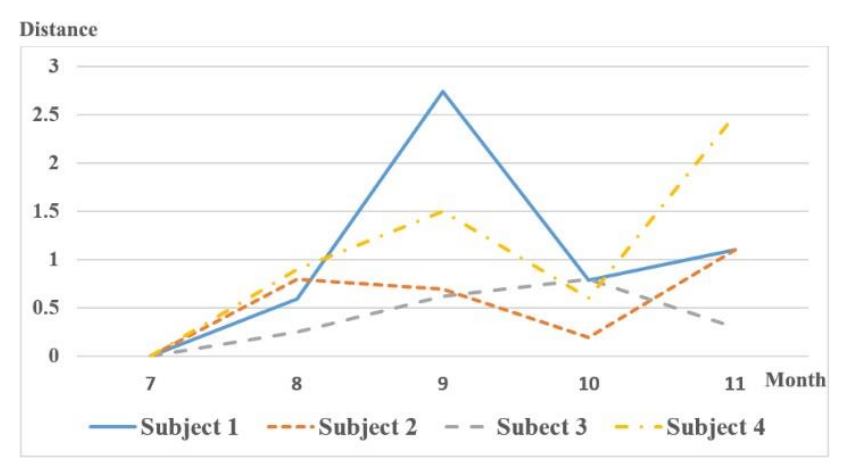

Fig. 7. Four subjects' density map dissimilarities comparison of five months using wearable device from histogram and texture features

\section{CONCLUSION AND FUTURE WORK}

Lifelogging physical activity (PA) monitoring and measurement is increasingly crucial task to the general public especially to the patients. Effectively assessing long term physical activities with mobile devices are becoming one of the most popular research areas in recent years. This paper investigates existing commercial mobile PA trackers from a mobile personalised healthcare platform MHA [2] using Moves mobile app and Google Fit on Samsung Gear $\mathrm{S} 2$. We first use Ellipse fitting model to remove irregular uncertainties (IU), then density maps are created to represent monthly PA intensity visualisations. Histogram and texture features are extracted from the maps for measures of PA intensity dissimilarities. The results demonstrate that commercial devices exist uncertainties due to a variety of reasons, while wearable devices exhibits more stable fluctuation than mobile phones which may further explored for healthcare studies.

\section{Reference}

[1] Moves, (n.d.). https://www.moves-app.com/ (accessed October 14, 2014).

[2] MHA, (n.d.). http://www.myhealthavatar.eu/ (accessed October 10, 2014).

[3] C.J. Caspersen, K.E. Powell, G.M. Christenson, Physical activity, exercise, and physical fitness: definitions and distinctions for health-related research., Public Health Rep. 100 (1985) 126-131. doi:10.2307/20056429.

[4] M.A. Minor, J.E. Hewett, R.R. Webel, S.K. Anderson, D.R Kay, Efficacy of physical conditioning exercise in patients with rheumatoid arthritis and osteoarthritis, Arthritis Rheum. 32 (1989) 1396-1405. doi:10.1002/anr.1780321108.

[5] 07363394.pdf, (n.d.)

[6] H. Martín, A.M. Bernardos, J. Iglesias, J.R. Casar, Activity logging using lightweight classification techniques in mobile devices, Pers. Ubiquitous Comput. 17 (2013) 675-695. doi:10.1007/s00779-012-0515-4.

[7] M.W. Lee, A.M. Khan, T.S. Kim, A single tri-axial accelerometer-based real-time personal life log system capable of human activity recognition and exercise information generation, Pers. Ubiquitous Comput. 15 (2011) 887-898. doi:10.1007/s00779-011-0403-3.

[8] M. Lee, J. Kim, K. Kim, I. Lee, S.H. Jee, S.K. Yoo, Physical Activity Recognition Using a Single Tri-Axis Accelerometer, October. I (2009) 20-23.

[9] Y. Kwon, K. Kang, C. Bae, H. Chung, J.H. Kim, Lifelog Agent for Human Activity Pattern Analysis on Health Avatar Platform, 20 (2014) 69-75.

[10] A. Jalal, Z. Uddin, T. Kim, Depth Video-based Human Activity Recognition System Using Translation and Scaling Invariant Features for Life Logging at Smart Home, (2012) 863-871.

[11] A.I. Journal, E. Berry, N. Kapur, L. Williams, S. Hodges, G. Smyth, J. Srinivasan, R. Smith, B. Wilson, E. Berry, N. Kapur, 
L. Williams, S. Hodges, G. Smyth, J. Srinivasan, R. Smith, B. Wilson, K.W. The, The use of a wearable camera, SenseCam, as a pictorial diary to improve autobiographical memory in a patient with limbic encephalitis: A preliminary report, 2011 (2011). doi:10.1080/09602010601029780.

[12] Fitbit Flex, (n.d.). http://www.fitbit.com/uk (accessed October 10, 2014).

[13] Nike+ Fuelband, (n.d.). http://www.nike.com/gb/en_gb/c/nikeplus-fuelband (accessed October 10, 2014)

[14] JawboneUp, (n.d.). https://jawbone.com/up (accessed February 10, 2014).

[15] Google Fit, (n.d.). https://www.google.com/fit/ (accessed April 14, 2014).

[16] a. Godfrey, a. K. Bourke, G.M. Ólaighin, P. van de Ven, J. Nelson, Activity classification using a single chest mounted triaxial accelerometer, Med. Eng. Phys. 33 (2011) 1127-1135. doi:10.1016/j.medengphy.2011.05.002.

[17] I.C. Gyllensten, A.G. Bonomi, Identifying types of physical activity with a single accelerometer: Evaluating laboratorytrained algorithms in daily life, IEEE Trans. Biomed. Eng. 58 (2011) 2656-2663. doi:10.1109/TBME.2011.2160723.

[18] D. Naranjo-Hernández, L.M. Roa, J. Reina-Tosina, M.Á. Estudillo-Valderrama, SoM: A smart sensor for human activity monitoring and assisted healthy ageing, IEEE Trans. Biomed. Eng. 59 (2012) 3177-3184. doi:10.1109/TBME.2012.2206384.

[19] A.M. Khan, Y.K. Lee, S. Lee, T.S. Kim, Accelerometer's position independent physical activity recognition system for long-term activity monitoring in the elderly, Med. Biol. Eng. Comput. 48 (2010) 1271-1279. doi:10.1007/s11517-010-07013.

[20] B. Perriot, J. Argod, J.L. Pepin, N. Noury, Characterization of Physical Activity in COPD Patients: Validation of a Robust Algorithm for Actigraphic Measurements in Living Situations, IEEE J. Biomed. Heal. Informatics. 18 (2014) 1225-1231. doi:10.1109/JBHI.2013.2282617.

[21] Z. Deng, P. Yang, Y. Zhao, X. Zhao, F. Dong, Life-Logging Data Aggregation Solution for Interdisciplinary Healthcare Research and Collaboration, 2015 IEEE Int. Conf. Comput. Inf. Technol. Ubiquitous Comput. Commun. Dependable, Auton. Secur. Comput. Pervasive Intell. Comput. (2015) 2315-2320. doi:10.1109/CIT/IUCC/DASC/PICOM.2015.342.

[22] and Z.D. J. Qi, P. Yang, D. Fan, A Survey of Physical Activity Monitoring and Assessment using Internet of Things Technology, in: 2015 IEEE Int. Conf. Comput. Inf. Technol. Ubiquitous Comput. Commun. Dependable, Auton. Secur. Comput. Pervasive Intell. Comput., IEEE, 2015: pp. 23532358.

[23] P. Yang, M. Hanneghan, J. Qi, Z. Deng, F. Dong, D. Fan, Improving the Validity of Lifelogging Physical Activity Measures in an Internet of Things Environment, 2015 IEEE Int. Conf. Comput. Inf. Technol. Ubiquitous Comput. Commun. Dependable, Auton. Secur. Comput. Pervasive Intell. Comput. (2015) 2309-2314. doi:10.1109/CIT/IUCC/DASC/PICOM.2015.341.

[24] H.-L. Chan, S.-C. Fang, Y.-L. Ko, M.-A. Lin, H.-H. Huang, C.H. Lin, Heart rate variability characterization in daily physical activities using wavelet analysis and multilayer fuzzy activity clustering., IEEE Trans. Biomed. Eng. 53 (2006) 133-139. doi:10.1109/TBME.2005.859811.

[25] Ó.D. Lara, A.J. Prez, M. a. Labrador, J.D. Posada, Centinela: A human activity recognition system based on acceleration and vital sign data, Pervasive Mob. Comput. 8 (2012) 717-729. doi:10.1016/j.pmcj.2011.06.004.

[26] K. Norton, L. Norton, D. Sadgrove, Position statement on physical activity and exercise intensity terminology $\leftleftarrows, 13$ (2010) 496-502. doi:10.1016/j.jsams.2009.09.008.

[27] J. Qi, P. Yang, M. Hanneghan, S. Tang, Neurocomputing Multiple density maps information fusion for effectively assessing intensity pattern of lifelogging physical activity, $\begin{array}{llll}\text { Neurocomputing. } & 220 & \text { (2017) } & 199-209 .\end{array}$ doi:10.1016/j.neucom.2016.06.073.

[28] J. Qi, P. Yang, M. Hanneghan, D. Fan, Z. Deng, F. Dong, Ellipse fitting model for improving the effectiveness of lifelogging physical activity measures in an Internet of Things environment, (2016) 107-113. doi:10.1049/iet-net.2015.0109.

[29] G. Blumchent, Metabolic Equivalents ( METS ) in Exercise Testing , Exercise Prescription , and Evaluation of Functional Capacity, 565 (1990) 555-565.

[30] M. Shoaib, S. Bosch, O. Durmaz Incel, H. Scholten, P.J.M.
Havinga, A Survey of Online Activity Recognition Using Mobile Phones, Sensors. 15 (2015) 2059-2085. doi:10.3390/s150102059. 